\title{
Association between cardiac autonomic function and physical activity in patients at high risk of sudden cardiac death: a cohort study
}

\author{
Xuerong Sun ${ }^{1}$, Shuang Zhao ${ }^{1}$, Keping Chen ${ }^{1}$, Wei Hua', Yangang Su², Xin Liư ${ }^{3}$, Wei Xü ${ }^{4}$, Fang Wang ${ }^{5}$,
} Xiaohan Fan', Yan Dai ${ }^{1}$, Zhimin Liu' and Shu Zhang ${ }^{1 *}$

\begin{abstract}
Background: High levels of physical activity (PA) and heart rate variability (HRV) are associated with cardiovascular benefits in patients with cardiovascular diseases. HRV, representing cardiac autonomic function, is positively associated with PA. However, the impacts of PA and cardiac autonomic function on cardiovascular outcomes were not analysed in the same study population. This lack of evidence supported our hypothesis that PA might contribute to cardiovascular benefits via enhanced cardiac autonomic function.
\end{abstract}

Methods: Patients with implantable cardioverter defibrillator (ICD) or cardiac resynchronisation therapy defibrillator (CRT-D) implantation were included from the SUMMIT registry. HRV and PA values were assessed during the first 30-60 days post device implantation using a continuous home monitoring system. Causal mediation analysis was conducted to explore the possible mediation function of HRV in the association of PA with long-term cardiac death and all-cause mortality in patients at a high risk of sudden cardiac death.

Results: Over a mean follow-up period of 47.7 months, 63 cardiac deaths (18.9\%) and 85 all-cause death events (25.5\%) were observed among 342 patients with ICD/CRT-D implantation. A positive linear association between HRV and PA was demonstrated and the $\beta$ value of HRV was 0.842 (95\% confidence interval [Cl]: $0.261-1.425, P=0.005$ ) in the multiple linear regression analysis. Multivariable Cox proportional hazards analysis revealed that high levels of PA $(\geq 11.0 \%)$ and $H R V(\geq 75.9 \mathrm{~ms})$ were independent protective factors against cardiac death (PA: hazard ratio $[H R]=0.273 ; 95 \% \mathrm{Cl}, 0.142-0.526, P<0.001 ; \mathrm{HRV}: \mathrm{HR}=0.224 ; 95 \% \mathrm{Cl}, 0.103-0.489, \mathrm{P}<0.001)$ and all-cause mortality (PA: HR=0.299; 95\% Cl, 0.177-0.505, P<0.001; HRV: HR=0.394; 95\% Cl, 0.231-0.674, P=0.001). Causal mediation analysis demonstrated partial mediation effects of PA that were mediated through HRV on cardiac death (mediation proportion $=12.9,95 \% \mathrm{Cl}: 2.2-32.0 \%, P=0.006)$ and all-cause mortality (mediation proportion $=8.2,95 \% \mathrm{Cl}: 1.6-20.0 \%$, $P=0.006)$.

Conclusions: HRV might be a modest mediator in the association between high levels of PA and the reduced risks of cardiac death and all-cause mortality in ICD/CRT-D recipients. This finding supports that enhanced cardiac autonomic function might be one of the underlying mechanisms by which regular PA contributes to cardiovascular benefits.

\footnotetext{
*Correspondence: zhangshufw@163.com

${ }^{1}$ Arrhythmia Center, State Key Laboratory of Cardiovascular Disease, Fuwai Hospital, National Center for Cardiovascular Diseases, Chinese

Academy of Medical Sciences and Peking Union Medical College, 167 Bei

Li Shi Road, Xicheng District, Beijing 100037, China

Full list of author information is available at the end of the article
} permits use, sharing, adaptation, distribution and reproduction in any medium or format, as long as you give appropriate credit to the original author(s) and the source, provide a link to the Creative Commons licence, and indicate if changes were made. The images or other third party material in this article are included in the article's Creative Commons licence, unless indicated otherwise in a credit line to the material. If material is not included in the article's Creative Commons licence and your intended use is not permitted by statutory regulation or exceeds the permitted use, you will need to obtain permission directly from the copyright holder. To view a copy of this licence, visit http://creativecommons.org/licenses/by/4.0/. The Creative Commons Public Domain Dedication waiver (http://creativeco mmons.org/publicdomain/zero/1.0/) applies to the data made available in this article, unless otherwise stated in a credit line to the data. 
Keywords: Physical activity, Heart rate variability, Implantable cardioverter defibrillator, Cardiac death, All-cause mortality, Causal mediation analysis

\section{Introduction}

Physical activity (PA) refers to any bodily movement resulting from skeletal muscle action, which can reflect the status of energy expenditure or exercise intensity of an individual $[1,2]$. Previous studies have reported that high levels of PA can protect against cardiovascular adverse events, cardiovascular death, and all-cause mortality in individuals with or without cardiovascular diseases (CVDs) [3-5]. Moreover, the dose-response relationships between the extent of PA and the risks of cardiac death and all-cause mortality were assessed to determine the optimal beneficial intervals $[3,6,7]$. Consequently, appropriate exercise-based cardiac rehabilitation or exercise training was recommended for patients with coronary heart diseases or chronic heart failure (HF) [3]. However, the potential underlying mechanism through which regular PA contributed to cardiovascular benefits remains unclear.

PA was previously assessed using self-reported structured questionnaires; however, this assessment had certain biases and errors depending on the individuals' educational level and cognitive function $[8,9]$. In recent studies, the values of the objective accelerometer-measured PA have been widely recognised [10]. Objective accelerometer-derived PA has a positive association with heart rate variability (HRV) [11-13]. HRV is a non-invasive and easily measured parameter in a time-dominant or frequency-dominant form and reflects cardiac autonomic function [14, 15]. Dysfunction of the autonomic nervous system also plays a crucial role in the clinical course of CVDs, and low levels of HRV indices are associated with increased risks of cardiovascular adverse events and mortality [16, 17]. Moreover, available evidence implies that regular PA might protect against death partly by enhanced cardiac autonomic function [11]. Nevertheless, a lack of studies evaluates the associations among PA, HRV, and long-term mortality in the same study population. The possible mediation function of cardiac autonomic function in the association between PA and long-term mortality demands further discussion.

Cardiac implantable electronic devices, such as implantable cardioverter defibrillator (ICD) or cardiac resynchronisation therapy defibrillator (CRT-D), can provide daily PA and HRV data that can be routinely collected using a continuous remote home monitoring system [18]. Therefore, in the present study, we performed a causal mediation analysis of the data from ICD/CRT-D recipients and aimed to 1 ) explore the causal chains among PA, HRV, and long-term mortality, 2) evaluate the possible mediation function of $\mathrm{HRV}$ in the association between high levels of PA and reduced risks of cardiac death and all-cause mortality, and 3) derive some evidence supporting that PA might contribute to cardiovascular benefits via enhanced cardiac autonomic function.

\section{Methods}

\section{Study participants}

In the present study, we conducted a retrospective analysis using the archived home monitoring transmission data from the Study of Home Monitoring System Safety and Efficacy in Cardiac Implantable Electronic Deviceimplanted Patients (SUMMIT) registry [7]. Patients who underwent ICD or CRT-D implantation between May 2010 and April 2014 were included upon meeting the following criteria: 1) ICD or CRT-D implantation in accordance with the recommendations outlined in the current guideline [19]; 2) the equipment with a remote home monitoring system and initiation of continuous monitoring after implantation; 3) capacity to supply available data related to daily HRV and PA; 4) age of the patients $\geq 18$ years at implantation; and 5) life expectancy $>1$ year after device implantation. Exclusion criteria were as follows: 1) diagnosis of persistent, long-standing, or permanent atrial fibrillation (AF); 2) AF episodes occurring during the first $30-60$ days after implantation; 3 ) $>5 \%$ of atrial or ventricular pacing percentages during the window period of HRV measurement; 4) missing or incomplete home monitoring data; 5) loss to follow-up; 6) diagnosis of a malignant tumour; or 7) schedule for heart transplantation.

This study complied with the Declaration of Helsinki and was approved by the ethics committee of Fuwai Hospital (the chief institute) and all other participating organisations. All patients provided written informed consent before study participation.

\section{Collection of baseline characteristics}

Data regarding baseline characteristics, including demographic characteristics (sex, age at implantation, body mass index [BMI], ICD or CRT-D implantation, and New York Heart Association [NYHA] Class III-IV), echocardiographic characteristics (left ventricular ejection fraction [LVEF], left ventricular end-diastolic dimension [LVEDD]), comorbidities (hypertension, diabetic mellitus $[\mathrm{DM}]$, stroke, dilated cardiomyopathy $[\mathrm{DCM}]$, hypertrophic cardiomyopathy $[\mathrm{HCM}]$, ischaemic 
cardiomyopathy $[\mathrm{ICM}]$, valve disease, prior myocardial infarction [MI], percutaneous coronary intervention $[\mathrm{PCI}]$, coronary artery bypass grafting [CABG], prior paroxysmal AF, long QT syndrome [LQTS], and pre-implant syncope), and medication (angiotensin-converting enzyme inhibitors or angiotensin receptor blockers [ACEIs/ARBs], aldosterone antagonists, diuretics, statins, calcium channel blockers [CCBs], beta-blockers, amiodarone, and antiplatelets), were collected from medical records.

\section{Measurement of PA and HRV}

Daily PA and HRV were detected using ICD devices (Biotronik, Germany). HRV and PA at baseline were assessed during the first 30-60 days after the ICD/CRT-D implantation, in accordance with the recommendations of previous studies [20]. The average values of daily PA and HRV at baseline were calculated for each patient.

PA was measured using the devices' acceleration sensors. PA was expressed as a percentage of the active time per day when the recorded rates were higher than basic rates, where $20 \%$ indicated $4.8 \mathrm{~h}$ of daily $\mathrm{PA}$, with a resolution of $2 \mathrm{~s}$. The accuracy of these acceleration sensors has been validated using the treadmill exercise [21].

HRV was measured in a time domain analysis and quantified as the standard deviation (SD) of the normal P-P intervals (SDNN algorithm) over a 24-h period [11, 15]. High levels of HRV can represent better joint sympathetic and parasympathetic modulation of heart rate [14, 15]. To obtain the reliable results of HRV analysis, the average values of daily atrial and ventricular pacing percentages should be both $\leq 5 \%$ during the window period of HRV measurement [22, 23].

\section{Follow-up and clinical outcomes}

Data regarding PA and HRV were recorded and transmitted using a remote home monitoring system every day. If data transmission was disrupted, the clinical research coordinator contacted the patients or their family members and confirmed the patients' health conditions immediately. Regular telephonic interviews or clinic visits were conducted to collect the information about clinical outcomes during follow-up. Data regarding the date of death and cause of death were obtained from the death certificate provided by family members. The primary endpoint was cardiac death (ICD-10: I00 to I09, I11, I20 to I51), and the secondary endpoint was all-cause mortality.

\section{Grouping}

Based on the baseline PA tertiles, patients were categorized into three groups: PA tertile 1 (range, 1.1-7.8\%; $n=114$ ), PA tertile 2 (range, $7.8-13.0 \% ; n=114$ ), and PA tertile 3 (range, $13.0-33.3 \% ; \mathrm{n}=114$ ).

\section{Statistical analysis}

The main exposure variables included PA and HRV. Continuous variables are presented as means \pm SDs, and categorical variables are presented as numbers and percentages. Baseline characteristics were compared between groups using one-way analyses of variance for continuous variables and the chi-squared tests for categorical variables. Rates of cardiac death and all-cause mortality events were calculated, and the difference was compared using the chi-squared test.

A box plot was generated to display the distribution of HRV based on different PA levels at baseline, and a scatter plot was used to describe the association between PA and HRV. Simple and multiple linear regression models were used to further explore their linear relations (Fig. 1, Path a). Multiple linear regression analysis considered the impacts from potential confounding variables, including age at implantation, sex, BMI, LVEF, LVEDD, ICD or CRT-D implantation, NYHA Class, hypertension, DM, stroke, DCM, ICM, MI, PCI, pre-implant syncope, prior AF, uses of ACEIs/ARBs, diuretics, and aldosterone antagonists, which should maintain consistent with those in the multivariable Cox regression models.

Restricted cubic splines were used to flexibly model and visualize the associations between PA or HRV and hazard ratios (HRs) for long-term clinical outcomes, as well as to explore their cut-off values for cardiac death and all-cause mortality based on the inflection points demonstrated by the smooth curve fitting. Univariable and multivariable Cox proportional hazards models were used to evaluate the independent predictive values of PA and HRV for long-term cardiac death and all-cause mortality (Fig. 1, Path b and c). Either PA or HRV entered the univariable Cox regression models. Variables selected for in the multivariable analysis were those with a $P$ value of $<0.05$ in the univariable models and other potential confounders. Multivariable Cox regression model 1 was adjusted for age at implantation, sex, BMI, LVEF, LVEDD, ICD or CRT-D implantation, NYHA Class, hypertension, DM, stroke, DCM, ICM, MI, PCI, pre-implant syncope, prior AF, uses of ACEIs/ARBs, diuretics, and aldosterone antagonists. Multivariable Cox regression model 2 was adjusted for the above-mentioned confounders and additional HRV or PA. HRs and 95\% confidence intervals (CIs) were calculated to show the impact at each level. A $P$ value $<0.05$ was considered significant, and all tests were two-sided.

Causal mediation analysis was conducted to assess the possible mediation function of HRV in the association between PA and long-term mortality. The mediation function represented the extent of PA that was able to influence the long-term mortality through modulating cardiac autonomic function. A causal mediation model 


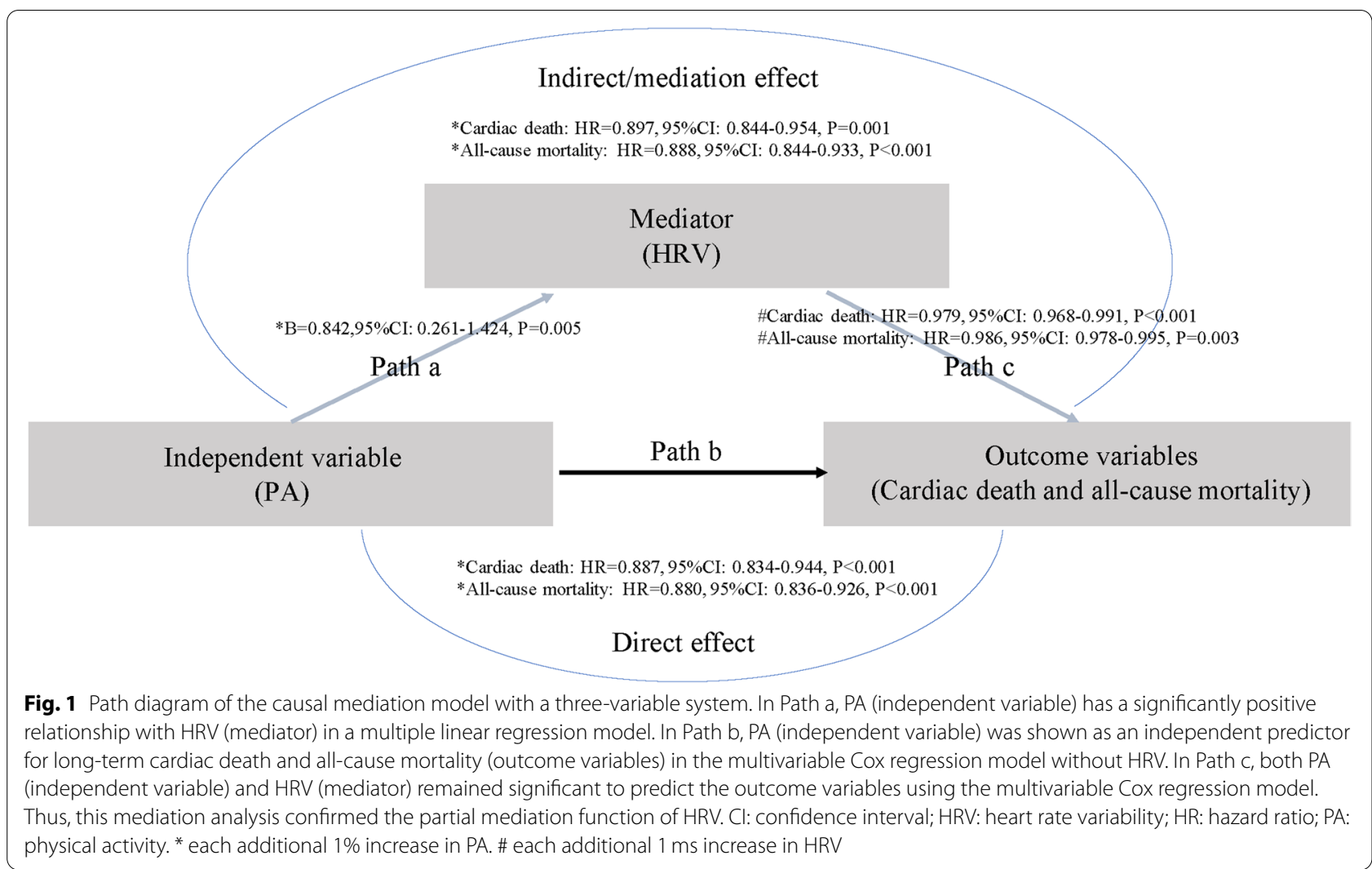

with a three-variable system was established following the Baron and Kenny's procedure [24, 25]. A path diagram is used to depict the causal chains (i.e., Path a-c) among PA (independent variable), HRV (mediator), and cardiac death and all-cause mortality (outcome variables) (Fig. 1). The mediation function is present when the following three conditions are met: a) PA is significantly associated with HRV in the multiple linear regression model (i.e., Path a, variations in the independent variable significantly accounted for the variations in presumed mediator); b) PA is shown as an independent predictor for cardiac death and all-cause mortality in the multivariable Cox regression model without HRV (i.e., Path b, variations in the independent variable significantly account for the outcome variables); c) HRV can independently predict the risks of cardiac death and all-cause mortality using the multivariable Cox regression model in which PA and HRV are simultaneously included (i.e., Path c, variations in presumed mediator significantly account for the outcome variables).

In Path c, if the effect of PA on clinical outcomes completely disappears, HRV fully mediates the association between PA and outcomes (full mediation), indicating that HRV as a single, dominant mediator; if the effect of PA still exits, HRV partially mediates the association between PA and outcomes (partial mediation), indicating the operation of multiple mediating factors. In addition, the $\mathrm{R}$ mediation package was used to estimate the average causal mediation effect (ACME) and the mediation proportion (prop. mediated) of PA on long-term mortality mediated through HRV [26]. The quasi-Bayesian Monte Carlo method was used to calculate the point estimates, 95\% CI values and $P$ values [27].

Statistical analyses were conducted using SPSS Statistics version 23.0 (IBM Corp., Armonk, NY) and R version 4.0.3 (Bunny-Wunnies Freak Out, The R Foundation for Statistical Computing, Vienna, Austria).

\section{Results \\ Baseline characteristics}

In this retrospective analysis, 342 out of 1008 patients with HRV and PA data were included. Patients were excluded due to lack of continuous HRV data availability with single-chamber ICDs $(n=509)$, incomplete HRV data $(n=60)$, incomplete PA data $(n=14)$, diagnosis of persistent, long-standing persistent $\mathrm{AF}, \mathrm{AF}$ episodes occurring during the first 30-60 days $(n=45)$, and $>5 \%$ of average daily atrial or ventricular pacing percentages $(n=35)$, or period of $<3$ months after device implantation $(n=3)$. Figure 2 shows the flow chart for patient selection. 


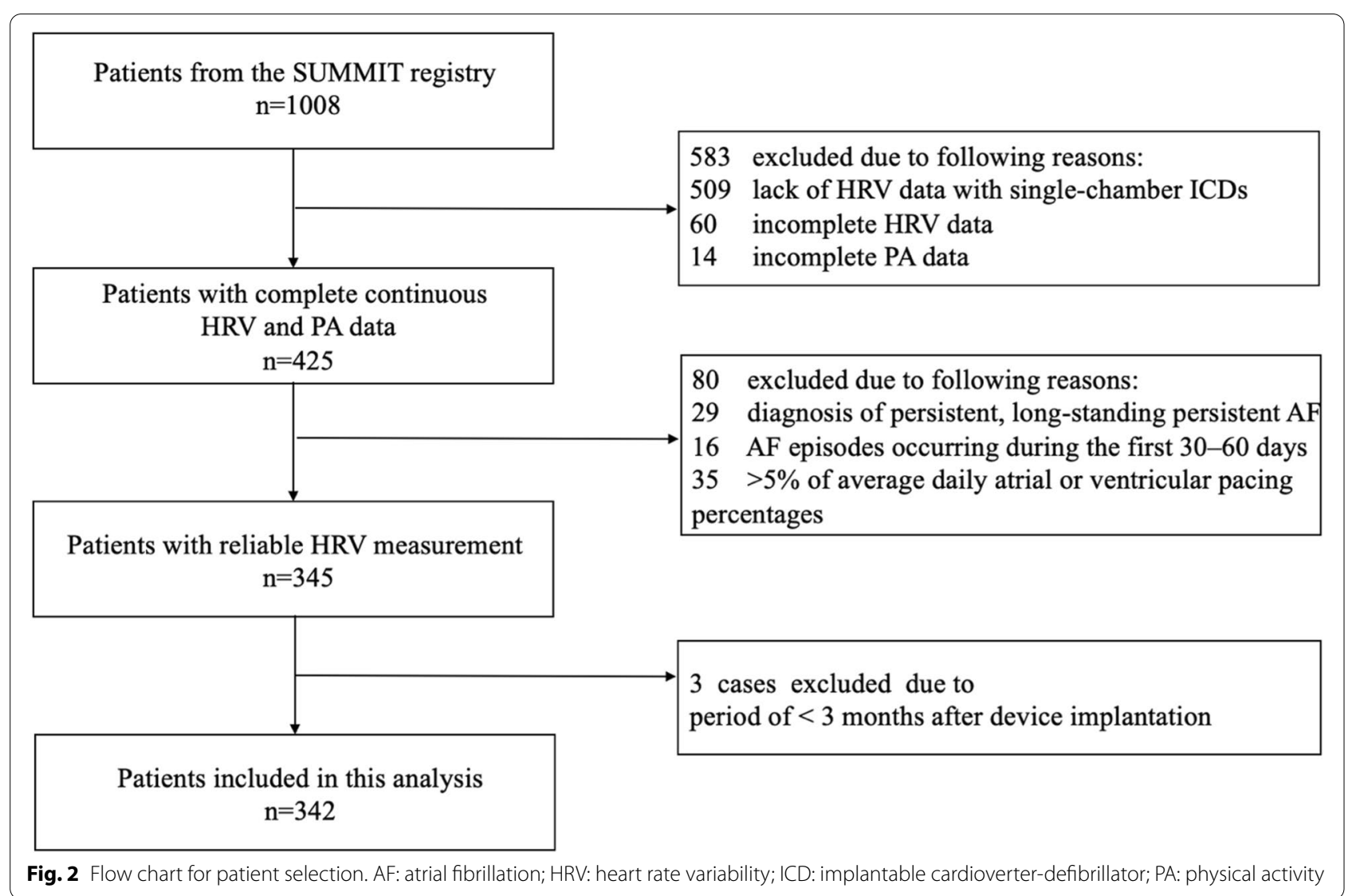

The mean age at implantation was $62.5 \pm 13.4$ years, and the male sex was dominant in the study cohort (78.1\%). CRT-Ds were implanted in 184 (53.8\%) patients and the mean LVEF was $39.13 \pm 14.43 \%$. The mean PA and HRV were $10.70 \pm 5.46 \%$ and $72.52 \pm 27.07 \mathrm{~ms}$, respectively. Significant differences in PA $(P<0.001)$, HRV $(\mathrm{P}<0.001)$, age at implantation $(\mathrm{P}<0.001)$, hypertension $(P=0.003)$, stroke $(P=0.001), \operatorname{ICM}(P=0.021), \mathrm{PCI}(\mathrm{P}<0.001)$ and CCBs usage $(P=0.019)$ were observed among different levels of baseline PA. Table 1 illustrates the comparison of baseline characteristics across three groups with different baseline PA levels.

\section{Clinical outcomes}

Over a mean follow-up duration of $47.7 \pm 20.8$ months, 62 cardiac deaths (18.1\%) and 94 all-cause mortality events $(27.5 \%)$ were observed. As baseline PA increased from tertile 1 to tertile 2 to tertile $3(5.18 \pm 2.01 \%$ vs. $10.30 \pm 1.48 \%$ vs. $16.93 \pm 3.19 \%)$, the corresponding HRV increased continuously $(62.40 \pm 25.43 \mathrm{~ms}$ vs. $72.23 \pm 26.87 \mathrm{~ms}$ vs. $78.90 \pm 28.25 \mathrm{~ms})$, but the incidence rates of cardiac death $(26.3 \%$ vs. $18.4 \%$ vs. $9.6 \%$, $P=0.005)$ and all-cause mortality $(42.1 \%$ vs. $29.8 \%$ vs. $10.5 \%, P=0.001)$ decreased continuously.

\section{Relationship between PA and HRV}

The distribution of HRV among groups with different PA levels is displayed using the box plot (Fig. 3a), with HRV increasing from PA tertile 1 group (HRV, median $=60.4 \mathrm{~ms}$, interquartile range $[\mathrm{IQR}]$ : $46.5-$ $75.8 \mathrm{~ms}$ ) to PA tertile 2 group (HRV, median $=70.4 \mathrm{~ms}$, IQR: $54.3-88.5 \mathrm{~ms}$ ) to PA tertile 3 group (HRV, median $=80.7 \mathrm{~ms}$, IQR: $59.2-97.8 \mathrm{~ms}$ ). A positive linear association between PA and HRV is demonstrated using the scatter plot (Fig. 3b).

A simple linear regression model was used to further assess the linear association between PA and HRV, and the $\beta$ of HRV was 1.193 (95\%CI: $0.657-1.729, P<0.001$ ). After adjusting for potential confounding variables in the multiple linear regression analysis, the $\beta$ of HRV was 0.842 (95\%CI: $0.261-1.425, P=0.005)$, indicating that HRV was increased by $0.842 \mathrm{~ms}$ for each additional $1 \%$ of PA at baseline.

\section{Cutoff values of HRV and PA for predicting long-term outcomes}

The associations between PA or HRV and HRs for cardiac death and all-cause mortality are demonstrated using the smooth curve fitting (Fig. 4). The risks of cardiac death 
Table 1 Baseline clinical characteristics

\begin{tabular}{|c|c|c|c|c|c|}
\hline Parameters & Total $(n=342)$ & Tertile $1(n=114)$ & Tertile $2(n=114)$ & Tertile $3(n=114)$ & P value \\
\hline \multicolumn{6}{|l|}{ Home monitoring data } \\
\hline Baseline PA, \% & $10.80 \pm 5.35$ & $5.18 \pm 2.01$ & $10.30 \pm 1.48$ & $16.93 \pm 3.19$ & - \\
\hline Baseline HRV, ms & $71.14 \pm 27.63$ & $62.40 \pm 25.43$ & $72.23 \pm 26.87$ & $78.80 \pm 28.85$ & - \\
\hline \multicolumn{6}{|l|}{ Demographics } \\
\hline Gender, male & $78.4 \%(268)$ & $76.3 \%(87)$ & $74.6 \%(85)$ & $84.2 \%(96)$ & $P=0.169$ \\
\hline Age at implantation, years & $62.37 \pm 13.48$ & $66.32 \pm 13.66$ & $63.26 \pm 13.07$ & $57.52 \pm 12.26$ & $P<0.001$ \\
\hline $\mathrm{BMI}, \mathrm{Kg} / \mathrm{m}^{2}$ & $23.56 \pm 2.87$ & $23.62 \pm 2.85$ & $23.53 \pm 3.26$ & $23.72 \pm 2.45$ & $P=0.890$ \\
\hline CRT-D implantation & $53.8 \%(184)$ & $51.8 \%(59)$ & $57.9 \%(66)$ & $51.8 \%(59)$ & $P=0.562$ \\
\hline NYHA, Class III-IV & $61.4 \%(210)$ & $67.5 \%(77)$ & $61.4 \%(70)$ & $55.3 \%(63)$ & $P=0.163$ \\
\hline \multicolumn{6}{|l|}{ Echocardiography } \\
\hline LVEF, \% & $39.13 \pm 14.43$ & $38.64 \pm 14.61$ & $39.48 \pm 14.20$ & $39.29 \pm 14.60$ & $P=0.900$ \\
\hline LVEDD, mm & $62.38 \pm 13.20$ & $60.17 \pm 11.36$ & $62.76 \pm 13.69$ & $64.23 \pm 14.18$ & $P=0.066$ \\
\hline \multicolumn{6}{|l|}{ Comorbidities } \\
\hline Hypertension & $35.1 \%(120)$ & $39.5 \%(45)$ & $43.0 \%(49)$ & $22.8 \%(26)$ & $P=0.003$ \\
\hline $\mathrm{DM}$ & $14.6 \%(50)$ & $17.5 \%(20)$ & $14.0 \%(16)$ & $12.3 \%(14)$ & $P=0.519$ \\
\hline Stroke & $2.6 \%(9)$ & $7.0 \%(8)$ & $0.9 \%(1)$ & $0.0 \%(0)$ & $P=0.001$ \\
\hline DCM & $29.8 \%(102)$ & $28.9 \%(33)$ & $29.8 \%(34)$ & $30.7 \%(35)$ & $P=0.959$ \\
\hline $\mathrm{HCM}$ & $3.8 \%(13)$ & $0.9 \%(1)$ & $5.3 \%(6)$ & $5.3 \%(6)$ & $P=0.081$ \\
\hline ICM & $39.8 \%(136)$ & $47.7 \%(54)$ & $42.1 \%(48)$ & $29.8 \%(34)$ & $P=0.021$ \\
\hline Valve disease & $2.3 \%(8)$ & $3.5 \%(4)$ & $1.8 \%(2)$ & $1.8 \%(2)$ & $P=0.671$ \\
\hline Prior Ml & $14.0 \%(48)$ & $20.2 \%(23)$ & $12.3 \%(14)$ & $9.6 \%(11)$ & $P=0.059$ \\
\hline $\mathrm{PCl}$ & $11.7 \%(40)$ & $21.1 \%(24)$ & $9.6 \%(11)$ & $4.4 \%(5)$ & $P<0.001$ \\
\hline CABG & $1.8 \%(6)$ & $3.5 \%(4)$ & $0.0 \%(0)$ & $1.8 \%(2)$ & $P=0.060$ \\
\hline Prior paroxysmal AF & $7.6 \%(26)$ & $9.6 \%(11)$ & $7.0 \%(8)$ & $6.1 \%(7)$ & $P=0.582$ \\
\hline LQTS & $1.5 \%(5)$ & $0.9 \%(1)$ & $1.8 \%(2)$ & $1.8 \%(2)$ & $P=0.801$ \\
\hline Pre-implant syncope & $15.8 \%(54)$ & $19.3 \%(22)$ & $9.6 \%(11)$ & $18.4 \%(21)$ & $P=0.087$ \\
\hline \multicolumn{6}{|l|}{ Medication } \\
\hline ACEIs/ARBs & $41.5 \%(142)$ & $43.0 \%(49)$ & $43.9 \%(50)$ & $37.7 \%(43)$ & $P=0.596$ \\
\hline Diuretics & $33.0 \%(113)$ & $39.5 \%(45)$ & $31.6 \%(36)$ & $28.1 \%(32)$ & $P=0.172$ \\
\hline Aldosterone antagonists & $45.0 \%(154)$ & $51.8 \%(59)$ & $41.2 \%(47)$ & $42.1 \%(48)$ & $P=0.208$ \\
\hline CCBs & $10.2 \%(35)$ & $2.0 \%(7)$ & $5.6 \%(19)$ & $2.6 \%(9)$ & $P=0.019$ \\
\hline Statins & $22.8 \%(78)$ & $24.6 \%(28)$ & $28.1 \%(32)$ & $15.8 \%(18)$ & $P=0.075$ \\
\hline Beta-blockers & $57.6 \%(197)$ & $57.0 \%(65)$ & $57.9 \%(66)$ & $57.9 \%(66)$ & $P=0.988$ \\
\hline Amiodarone & $28.1 \%(96)$ & $27.2 \%(31)$ & $23.7 \%(27)$ & $33.3 \%$ (38) & $P=0.260$ \\
\hline Antiplatelets & $21.6 \%(74)$ & $27.2 \%(31)$ & $22.8 \%(26)$ & $14.9 \%(17)$ & $P=0.074$ \\
\hline
\end{tabular}

$A C E l s$ angiotensin-converting enzyme inhibitors, $A F$ atrial fibrillation, $A R B$ s angiotensin receptor blockers, $B M I$ body mass index, $C A B G$ coronary artery bypass grafting, $C C B$ calcium channel blockers, $C R T-D$ cardiac resynchronization therapy with defibrillation, DCM dilated cardiomyopathy, DM diabetes mellitus, HCM hypertrophic cardiomyopathy, HRV heart rate variability, ICM ischemic cardiomyopathy, LQTS long QT syndrome, LVEF left ventricular ejection fraction, LVEDD left ventricular enddiastolic dimension, $M I$ myocardial infarction, $P A$ physical activity, $P C l$ percutaneous coronary intervention

and all-cause mortality declined rapidly before $75.9 \mathrm{~ms}$ of HRV and $11.0 \%$ of PA and then started to decrease slowly thereafter. Thus, $75.9 \mathrm{~ms}$ of HRV and $11.0 \%$ of PA were obtained as the cut-off values.

\section{Predictive values of PA for long-term outcomes}

Univariable and multivariable Cox regression analyses revealed that increased PA protected against the risks of cardiac death and all-cause mortality (Table 2). In multivariable Cox regression model 1, a high level of PA $(\geq 11.0 \%)$ was associated with reduced risks of cardiac death $(\mathrm{HR}=0.239 ; 95 \% \mathrm{CI}: 0.124-0.460, P<0.001)$ and all-cause mortality $(\mathrm{HR}=0.277 ; 95 \% \mathrm{CI}$ : $0.163-0.469$, $\mathrm{P}<0.001)$ after adjusting for age at implantation, sex, BMI, LVEF, LVEDD, ICD or CRT-D implantation, NYHA Class, hypertension, DM, stroke, DCM, ICM, MI, PCI, pre-implant syncope, prior AF, use of ACEIs/ARBs, use of diuretics, and use of aldosterone antagonists. When 


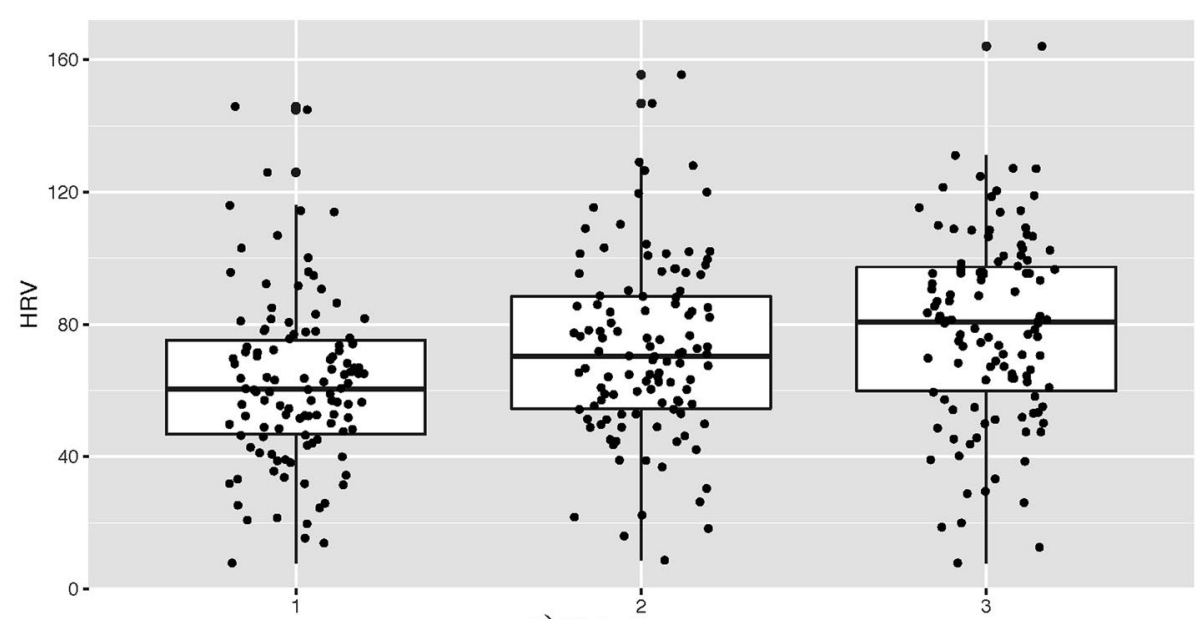

a) PA Tertile group

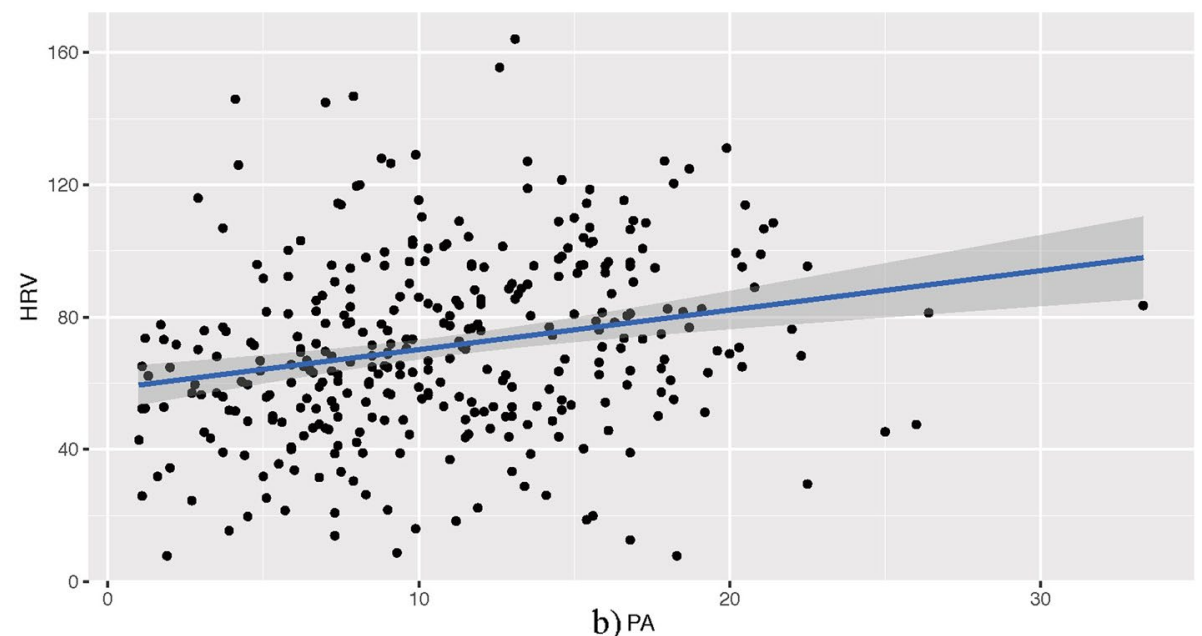

Fig. 3 Box and scatter plots of the association between PA and HRV. HRV, heart rate variability; PA, physical activity

adjusting for the above-mentioned variables and additional HRV, the predictive values of PA remained significant for cardiac death $(\mathrm{HR}=0.897$; $95 \%$ CI: $0.844-0.954$, $P=0.001)$ and all-cause mortality $(\mathrm{HR}=0.888 ; 95 \% \mathrm{CI}$ : 0.844-0.933, $\mathrm{P}<0.001$ ) in multivariable model 2 , indicating that each $1 \%$ increase in PA could result in a 10.3 and $11.2 \%$ reduction in the risks of cardiac death and allcause mortality, respectively.

\section{Predictive values of HRV for long-term outcomes}

In 342 ICD/CRT-D recipients, univariable and multivariable Cox regression analyses showed that HRV was inversely associated with the incidence rates of cardiac death and all-cause mortality (Table 3). Multivariable Cox regression analyses revealed that a high level of HRV $(\geq 75.9 \mathrm{~ms})$ was associated with reduced risks of cardiac death $(\mathrm{HR}=0.197 ; 95 \% \mathrm{CI}: 0.090-0.428, P<0.001)$ and all-cause mortality ( $\mathrm{HR}=0.351 ; 95 \% \mathrm{CI}: 0.205-0.600$, $\mathrm{P}<0.001)$ when adjusted for age at implantation, sex,
BMI, LVEF, LVEDD, ICD or CRT-D implantation, NYHA Class, hypertension, DM, stroke, DCM, ICM, MI, PCI, pre-implant syncope, prior AF, use of ACEIs/ ARBs, use of diuretics, and use of aldosterone antagonists. After adjusting for the above-mentioned variables and additional PA in multivariable model 2, the predictive values of HRV remained significant for cardiac death $(\mathrm{HR}=0.979 ; 95 \% \mathrm{CI}$ : $0.968-0.991, \mathrm{P}<0.001)$ and all-cause mortality (HR $=0.986$; 95\% CI: $0.978-0.995$, $P=0.003$ ), indicating that each $1 \mathrm{~ms}$ increase in HRV could result in a 2.1 and $1.4 \%$ reduction in the risks of cardiac death and all-cause mortality, respectively.

\section{Mediation analysis}

Figure 1 summarises the results of causal mediation analysis. In Path a, PA (independent variable) had a positive linear relationship with HRV (mediator) in a multiple linear regression analysis. In Path $b$, when HRV was 

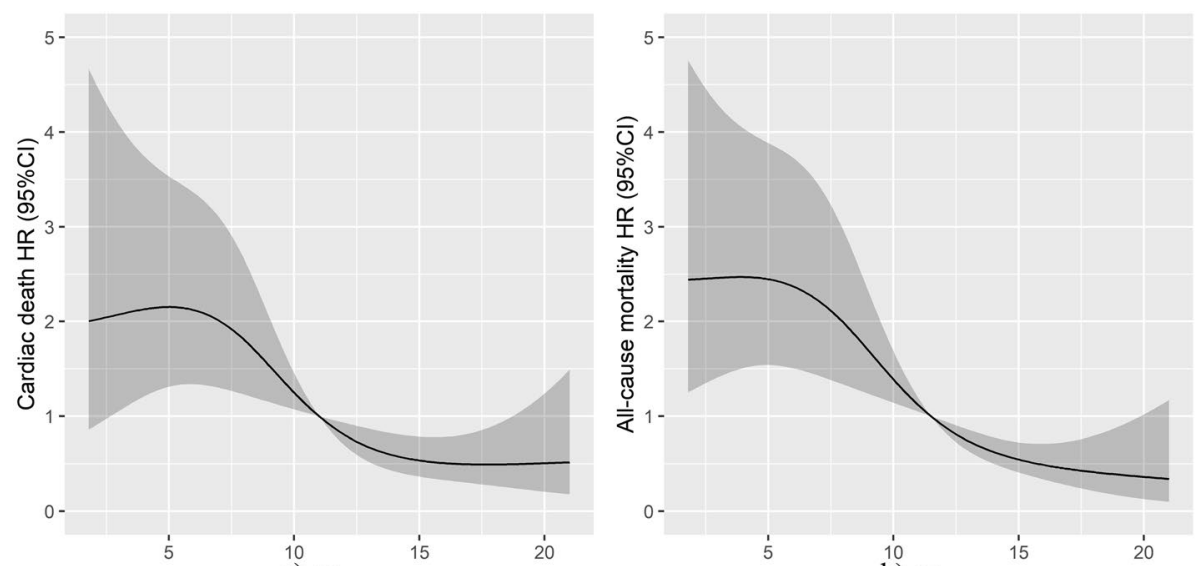

a) PA
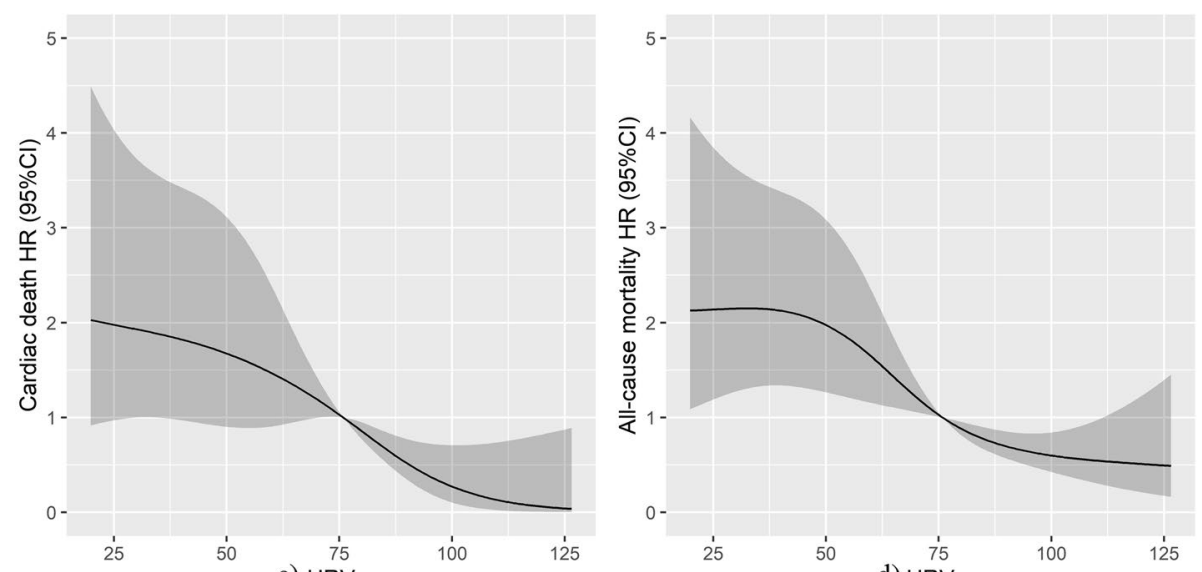

c) HRV

d) HRV

Fig. 4 Relationships between HRV/PA and HRs for cardiac death and all-cause mortality. 3a) smoothing curve fitting of PA in predicting the risks of cardiac death; 3b) smoothing curve fitting of PA in predicting the risks of all-cause mortality; 3c) smoothing curve fitting of HRV in predicting the risks of cardiac death; $3 \mathrm{~d}$ ) smoothing curve fitting of HRV in predicting all-cause mortality. HR, hazard ratio; HRV, heart rate variability; PA, physical activity

Table 2 Predictive values of PA for long-term mortality

\begin{tabular}{|c|c|c|c|c|c|c|}
\hline & \multicolumn{2}{|c|}{ Univariable Cox regression model } & \multicolumn{2}{|c|}{$\begin{array}{l}\text { Multivariable Cox regression } \\
\text { model } 1\end{array}$} & \multicolumn{2}{|c|}{$\begin{array}{l}\text { Multivariable Cox regression } \\
\text { model } 2\end{array}$} \\
\hline & $\mathrm{HR}(95 \% \mathrm{Cl})$ & $P$ value & $\mathrm{HR}(95 \% \mathrm{Cl})$ & $P$ value & $\mathrm{HR}(95 \% \mathrm{Cl})$ & P value \\
\hline \multicolumn{7}{|l|}{ Primary endpoint: cardiac death } \\
\hline Per $1 \% /$ increase & $0.904(0.858-0.952)$ & $P<0.001$ & $0.887(0.834-0.944)$ & $P<0.001$ & $0.897(0.844-0.954)$ & $P=0.001$ \\
\hline$\geq 11.0 \%$ group vs $<11.0 \%$ group & $0.280(0.154-0.509)$ & $P<0.001$ & $0.239(0.124-0.460)$ & $P<0.001$ & $0.273(0.142-0.526)$ & $P<0.001$ \\
\hline \multicolumn{7}{|c|}{ Secondary endpoint: all-cause mortality } \\
\hline Per $1 \% /$ increase ${ }^{a}$ & $0.886(0.848-0.926)$ & $P<0.001$ & $0.880(0.836-0.926)$ & $P<0.001$ & $0.888(0.844-0.933)$ & $P<0.001$ \\
\hline$\geq 11.0 \%$ group vs $<11.0 \%$ group & $0.276(0.170-0.449)$ & $P<0.001$ & $0.277(0.163-0.469)$ & $P<0.001$ & $0.299(0.177-0.505)$ & $P<0.001$ \\
\hline
\end{tabular}

Multivariable Cox regression model 1 was adjusted for age at implantation, sex, BMI, LVEF, LVEDD, ICD or CRT-D implantation, NYHA Class, hypertension, DM, stroke, $\mathrm{DCM}$, ICM, MI, PCl, pre-implant syncope, prior AF, use of ACEls/ARBs, use of diuretics, and use of aldosterone antagonists. Multivariate Cox regression model 2 was adjusted for the above-mentioned confounders and additional HRV

$A C E I S / A R B s$ angiotensin-converting enzyme inhibitors or angiotensin receptor blockers, $A F$ atrial fibrillation, $B M I$ body mass index, $C l$ confidence interval, $C R T$ - $D$ cardiac resynchronisation therapy defibrillator, $D M$ diabetic mellitus, $H R V$ heart rate variability, $H R$ hazard ratio, ICD implantable cardioverter defibrillator, $I C M$ ischaemic cardiomyopathy, LVEF left ventricular ejection fraction, LVEDD left ventricular end-diastolic dimension, MI myocardial infarction, NYHA New York Heart Association, PA physical activity, $P C I$ percutaneous coronary intervention

${ }^{a}$ each additional $1 \%$ increase in PA 
Table 3 Predictive values of HRV for long-term mortality

\begin{tabular}{|c|c|c|c|c|c|c|}
\hline & \multicolumn{2}{|c|}{$\begin{array}{l}\text { Univariable Cox regression } \\
\text { model }\end{array}$} & \multicolumn{2}{|c|}{$\begin{array}{l}\text { Multivariable Cox regression } \\
\text { model } 1\end{array}$} & \multicolumn{2}{|c|}{$\begin{array}{l}\text { Multivariable Cox regression } \\
\text { model } 2\end{array}$} \\
\hline & $\mathrm{HR}(95 \% \mathrm{Cl})$ & $P$ value & $\mathrm{HR}(95 \% \mathrm{Cl})$ & $P$ value & $\mathrm{HR}(95 \% \mathrm{Cl})$ & P value \\
\hline \multicolumn{7}{|l|}{ Primary endpoint: cardiac death } \\
\hline Per $1 \mathrm{~ms} /$ increase $^{\mathrm{a}}$ & $0.978(0.968-0.987)$ & $P<0.001$ & $0.978(0.967-0.989)$ & $P<0.001$ & $0.979(0.968-0.991)$ & $P<0.001$ \\
\hline$\geq 75.9$ ms group vs $<75.9$ ms group & $0.169(0.080-0.355)$ & $P<0.001$ & $0.197(0.090-0.428)$ & $P<0.001$ & $0.224(0.103-0.489)$ & $P<0.001$ \\
\hline \multicolumn{7}{|c|}{ Secondary endpoint: all-cause mortality } \\
\hline Per 1 ms/increase $e^{a}$ & $0.983(0.976-0.991)$ & $P<0.001$ & $0.984(0.976-0.993)$ & $P<0.001$ & $0.986(0.978-0.995)$ & $P=0.003$ \\
\hline$\geq 75.9$ ms group vs $<75.9 \mathrm{~ms}$ group & $0.291(0.176-0.482)$ & $P<0.001$ & $0.351(0.205-0.600)$ & $P<0.001$ & $0.394(0.231-0.674)$ & $P=0.001$ \\
\hline
\end{tabular}

Multivariable Cox regression model 1 was adjusted for age at implantation, sex, BMI, LVEF, LVEDD, ICD or CRT-D implantation, NYHA Class, hypertension, DM, stroke, $\mathrm{DCM}, \mathrm{ICM}, \mathrm{MI}, \mathrm{PCl}$, pre-implant syncope, prior AF, use of ACEls/ARBs, use of diuretics, and use of aldosterone antagonists. Multivariate Cox regression model 2 was adjusted for the above-mentioned confounders and additional PA

$A C E I S / A R B s$ angiotensin-converting enzyme inhibitors or angiotensin receptor blockers, $A F$ atrial fibrillation, $B M I$ body mass index, $C l$ confidence interval, $C R T-D$ cardiac resynchronisation therapy defibrillator, DM diabetic mellitus, HRV heart rate variability, $H R$ hazard ratio, ICD implantable cardioverter defibrillator, ICM ischaemic cardiomyopathy, LVEF left ventricular ejection fraction, LVEDD left ventricular end-diastolic dimension, MI myocardial infarction, NYHA New York Heart Association, PA physical activity, $\mathrm{PCl}$ percutaneous coronary intervention

a each additional $1 \mathrm{~ms}$ increase in HRV

not included in the multivariable Cox regression model, PA (independent variable) was an independent predictor for long-term cardiac death and all-cause mortality (outcome variables). In Path c, both PA (independent variable) and HRV (mediator) remained significant to predict the outcome variables in the multivariable Cox regression model. Thus, this mediation analysis confirmed the partial mediation function of HRV in the association between high levels of PA and increased risks of cardiac death and all-cause mortality.

Figure 5 shows that for cardiac death, the mediation effect was statistically significant $(\mathrm{ACME}=56.7,95 \% \mathrm{CI}$ : 7.4-148.7, $P=0.006$; total effect $=402.3$, 95\%CI: $197.3-$ 705.1, $P<0.001$ ), and $12.9 \%$ (prop. Mediated $=12.9$, 95\%CI: $2.2-32.0 \%, \mathrm{P}=0.006)$ of the mediation effect of PA on the risks of cardiac death was mediated through HRV. For all-cause mortality, the mediation effect remained significant $(\mathrm{ACME}=20.0,95 \% \mathrm{CI}$ : 2.9-45.6, $\mathrm{P}=0.006$; total effect $=225.2$, 95\%CI: $141.2-318.7$, $\mathrm{P}<0.001$ ), and $8.2 \%$ (prop. Mediated $=8.2,95 \% \mathrm{CI}$ : $1.6-$ $20.0 \%, P=0.006)$ of the mediation effect of PA on the risks of all-cause mortality was mediated through HRV.

\section{Discussion}

In this retrospective analysis, data regarding PA and HRV obtained from a continuous remote home monitoring system were included, and causal mediation analysis was established to explore the possible mediation function of HRV in the association between PA and long-term mortality in patients with a high risk of sudden cardiac death (SCD). The main findings were: 1 ) HRV was positively associated with PA, 2) both PA and HRV were independent protective predictors for cardiac death and all-cause mortality, 3) the causal mediation analysis confirmed partial mediation effects of PA on the risks of cardiac death and all-cause mortality among 342 ICD/CRT-D recipients; 12.9 and $8.2 \%$ of the mediation effects of PA on the risks of cardiac death and all-cause mortality, respectively, were mediated through HRV. These findings support that PA might contribute to cardiovascular benefits partly via enhanced cardiac autonomic function.

The autonomic nervous system plays a crucial role in the clinical course of CVDs through modulating arrhythmias, heart rate, blood pressure, breathing, digestion, etc. [14-17]. HRV is the fluctuation in the intervals between heartbeats, which can be acquired using the time-domain or frequency-domain measurement from ECG recordings [14, 15]. High levels of HRV can reflect better modulation between sympathetic and parasympathetic activities [14, 15]. Some studies conducted crosssectional analyses to investigate the association between cardiac autonomic function and PA in the general population at different ages $[12,13,28,29]$. Soares-Miranda et al. obtained HRV indices from a 24-h Holter in 985 older adults in the Cardiovascular Health Study [28]. Data regarding PA, which was categorised as leisuretime activity, walking distance, and walking pace, were obtained using the modified Minnesota PA questionnaire. Greater PA demonstrated an association with more favourable HRV indices in older adults. In addition, the Finnish MOPD study and a British study of civil servants focused on 3629 adolescent men and 3328 middleaged (35-55 years) people, respectively [12, 29]. HRV was derived from 5-min ECG recordings. Moderate and vigorous PA were observed associated with higher HRV indices in both studies. In the above-mentioned studies, 


\section{Effects of PA on cardic death mediated through HRV}

Prop. Mediated $=12.9 \%, 95 \% \mathrm{Cl}: 2.2 \%-32.0 \%, P=0.006$

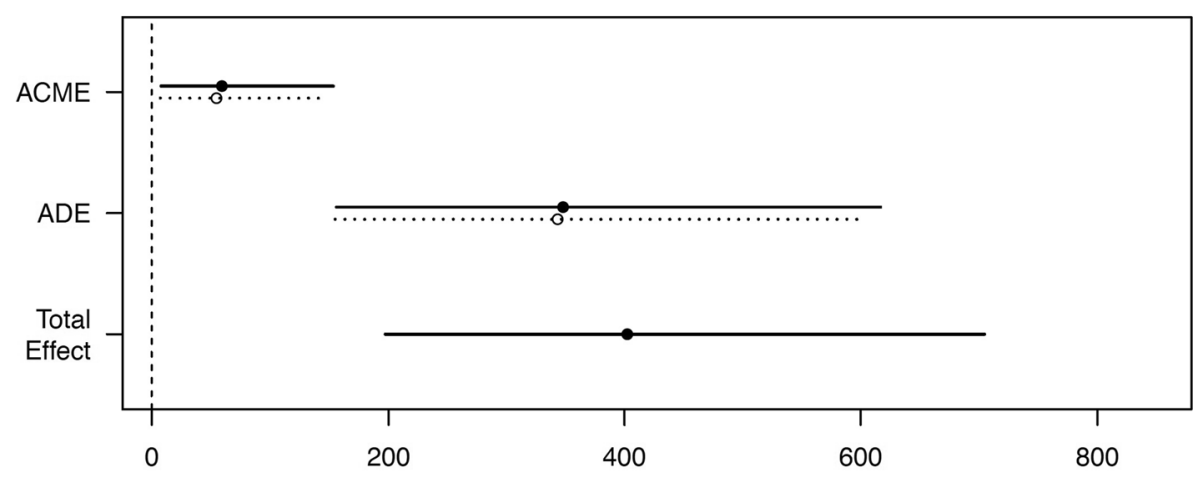

Effects of PA on all-cause mortality mediated through HRV

Prop. Mediated $=8.2 \%, 95 \% \mathrm{Cl}: 1.6 \%-20.0 \%, \mathrm{P}=0.006$

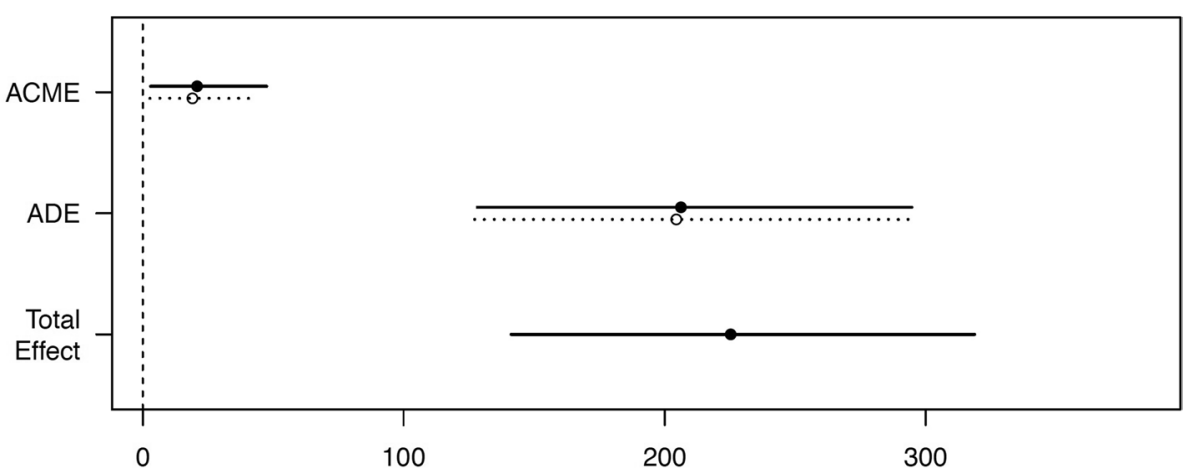

Fig. 5 Causal mediation analysis results. ACME stands for average causal mediation effects of PA on the long-term mortality mediated through HRV; ADE stands for average direct effects of PA on the long-term mortality; Total effect stands for the total effects (direct and indirect) of PA on the long-term mortality; Prop. Mediated describes the proportion of the indirect/mediation effect of PA on the long-term mortality that goes through the mediator. ACME, average causal mediation effect; $\mathrm{ADE}$, average direct effect; $\mathrm{Cl}$, confidence interval; $\mathrm{HRV}$, heart rate variability; PA, physical activity; Prop. Mediated: mediation proportion

HRV values was acquired from traditional short-term ECG recordings, or PA was obtained from self-assessment questionnaires [12, 13, 28, 29]. Differently, Zhao S et al. obtained the objective accelerometer-measured PA and time-dominant HRV values from continuous ECG recordings in ICD/CRT-D recipients, and confirmed a significant correlation $(\mathrm{r}=0.601, P<0.001)$ between PA and HRV although the sample size was small [11]. In the present study, both HRV and PA values were derived from continuous and quantitative monitoring data and their average daily values were measured during the first 30-60 days after ICD/CRT-D implantation for each patient, as recommended by published evidence $[11,20]$. Box and scatter plot analyses, and multiple linear regression analysis were conducted to further investigate the association between PA and HRV. The results showed PA had a robust positive linear relationship with HRV, which was consistent with previous findings. Therefore, the present study provided more evidence to support that regular physical exercise was related to enhanced cardiac autonomic regulation. Although the cellular and molecular interpretations of the positive linear relationship between cardiac autonomic and PA are complex, PA may modulate cardiac autonomic function by improving cardiomyocyte contractile capacity and cardiac electrical stability [30,31].

Previous studies have clarified the predictive values of HRV or PA for long-term outcomes in patients with different CVDs [3-7, 16, 17, 20, 32-34]. Whether PA was self-reported or accelerometer-derived, low levels of PA were associated with increased incidences of hospitalisations for HF, cardiovascular adverse events, cardiovascular death, and all-cause mortality [3-7, 20]. In previous studies on different populations, HRV values were derived from 10-s to 24-h ECG recordings [16, 17]. A low level of HRV, indicative of cardiac autonomic 
dysfunction, was identified as an independent risk factor for ventricular arrhythmia in patients who had experienced acute MI and in those who were at a high risk of SCD $[16,17,32]$. Other studies have reported similar results for cardiac death and all-cause mortality in older adults and patients at a high risk of type 2 DM [33, 34]. However, the predictive values of PA and HRV for longterm mortality were not analysed in the same study population. Moreover, the mediation function of improved HRV in the relationship between regular PA and satisfying clinical outcomes was not explored. Schwartz et al. observed that 6 weeks of treadmill training reduced the incidence of ventricular fibrillation during acute myocardial ischaemia by $100 \%$, while HRV increased by $74 \%$ derived from 25-min ECG recordings [35]. It implied that the underlying mechanism of $100 \%$ survival rate might be that exercise training improved cardiac autonomic modulation [35]. In the present study, the association between PA and HRV, and their effects on cardiac death and allcause mortality were simultaneously analysed in ICD/ CRT-D recipients. Additionally, we conducted a causal mediation analysis to explore the possible mediation function of cardiac autonomic function in the association between PA and long-term cardiac death and all-cause mortality.

In the multivariable Cox regression analysis, both HRV and PA were found to be independent predictors for cardiac death and all-cause mortality, regardless of various baseline characteristics. Every additional 1\% increase in PA could result in a 10.3 and $11.2 \%$ reduction in risks of cardiac death and all-cause mortality, respectively; every additional $1 \mathrm{~ms}$ increase in HRV could result in a 2.1 and $1.4 \%$ reduction in risks of cardiac death and all-cause mortality, respectively. The partial mediation function was confirmed using the causal mediation analysis following the Baron and Kenny's procedure [24]. It was further estimated that the proportions of indirect effects of PA on the risks of cardiac death and all-cause mortality mediated through cardiac autonomic nervous modulation were 12.9 and $8.2 \%$, respectively. This finding revealed that the underlying mechanisms were multifactorial and complex, and enhanced cardiac autonomic regulation might be one of the most important mechanisms by which regular PA contributed to cardiovascular benefits. Cardiac autonomic regulation should be emphasised in the management of patients after ICD/ CRT-D implantation. More effective exercise regimens to enhance the cardiac autonomic function should be developed in the clinical practice. In contrast, the direct effects of PA, which probably include improved cardiac output, enhanced cardiorespiratory fitness, reduced systemic vascular resistance, and regulation on cardiac remodelling, etc., cannot be ignored [36].

\section{Limitations}

Two potential limitations were considered in the present study. First, in the present study, only observational data regarding PA and HRV from ICD/CRT-D recipients were retrospectively analysed. Interventional studies with a larger sample size are required to further explore the mediation function of HRV in the association between high levels of PA and long-term mortality in different populations. Second, selection bias can occur, and caution should be exercised while generalizing the results to populations other than ICD/CRT-D recipients. Third, HRV indices were acquired in a time-dominant form using SDNN algorithm. SDNN algorithm can allow for precise interpretations of cardiac autonomic function and no gold standards for HRV measurement exist [15]. However, it would be better if both frequency-dominant and time-dominant HRV indices could be evaluated.

\section{Conclusions}

Causal mediation analysis revealed partial mediation function of HRV in the association between PA and longterm mortality in ICD/CRT-D recipients. About 12.9 and $8.2 \%$ of the mediation effects of PA on the risks of cardiac death and all-cause mortality, respectively, were mediated through HRV. These findings indicated that enhanced cardiac autonomic function might be one of the underlying mechanisms by which regular PA contributed cardiovascular benefits.

\section{Abbreviations \\ ACEl: angiotensin-converting enzyme inhibitor; ACME: average causal mediation effect; ADE: average direct effect; AF: atrial fibrillation; ARB: angiotensin receptor blocker; BMI: body mass index; CABG: coronary artery bypass grafting; CCB: calcium channel blocker; Cl: confidence interval; CRT-D: cardiac resynchronisation therapy defibrillator; CVD: cardiovascular disease; DCM: dilated cardiomyopathy; DM: diabetes mellitus; ECG: electrocardiogra- phy; HCM: hypertrophic cardiomyopathy; HF: heart failure; HR: hazard ratio; HRV: heart rate variability; ICD: implantable cardioverter defibrillator; ICM: ischaemic cardiomyopathy; LVEDD: left ventricular end-diastolic dimension; LVEF: left ventricular ejection fraction; LQTS: long QT syndrome; MI: myocar- dial infarction; NYHA: New York Heart Association; PA: physical activity; PCl: percutaneous coronary intervention; Prop. Mediated: mediation proportion; SCD: sudden cardiac death; SD: standard deviation; SUMMIT: Study of Home Monitoring System Safety and Efficacy in Cardiac Implantable Electronic Device-implanted Patients.}

\section{Acknowledgements}

Not applicable.

\section{Authors' contributions}

Sun $X$ and Zhao Shuang contributed to the conception or design of the work. Sun X, Chen K, Hua W, Su Y, Liu X, Xu W, Wang F, Fan X, Dai Y and Liu Z contributed to the acquisition, analysis, or interpretation of data for the work. Sun $X$ drafted the manuscript. Zhang Shu critically revised the manuscript. The author(s) read and approved the final manuscript.

\section{Funding}

This study was supported by Natural Science Foundation of China (81470466) and the National Science \& Technology Pillar Program during the 12th FiveYear Plan Period (2011BAl11B02). The funding only provided financial support 
but had no role in study design, data collection, analysis, data interpretation, manuscript writing, or decision to submit the manuscript for publication.

\section{Availability of data and materials}

The datasets generated and analysed during the current study are not publicly available due to the Fuwai Hospital regulations but are available from the corresponding author on reasonable request.

\section{Declarations}

\section{Ethics approval and consent to participate}

This study was approved by the ethics committee of Fuwai Hospital (the chief institute, Approval No: 2010-296) and all other participating organisations. All patients provided written informed consent before entering this study.

\section{Consent for publication}

Not applicable.

\section{Competing interests}

The authors declare that they have no competing interests.

\section{Author details}

${ }^{1}$ Arrhythmia Center, State Key Laboratory of Cardiovascular Disease, Fuwai Hospital, National Center for Cardiovascular Diseases, Chinese Academy of Medical Sciences and Peking Union Medical College, 167 Bei Li Shi Road, Xicheng District, Beijing 100037, China. ${ }^{2}$ Department of Cardiology, Shanghai Institute of Cardiovascular Diseases, Zhongshan Hospital, Fudan University, Shanghai, China. ${ }^{3}$ Department of Cardiology, Beijing Anzhen Hospital, Capital Medical University, Beijing, China. ${ }^{4}$ Department of Cardiology, Nanjing Drum Tower Hospital, Nanjing, China. ${ }^{5}$ Department of Cardiology, Shanghai First People's Hospital, Shanghai Jiao Tong University School of Medicine, Shanghai, China.

Received: 14 March 2021 Accepted: 6 September 2021

Published online: 20 September 2021

\section{References}

1. Haskell WL, Lee IM, Pate RR, Powell KE, Blair SN, Franklin BA, et al. Physical activity and public health: updated recommendation for adults from the American College of Sports Medicine and the American Heart Association. Med Sci Sports Exerc. 2007;39(8):1423-34.

2. Hills AP, Mokhtar N, Byrne NM. Assessment of physical activity and energy expenditure: an overview of objective measures. Front Nutr. 2014;1:5.

3. Jeong SW, Kim SH, Kang SH, Kim HJ, Yoon CH, Youn TJ, et al. Mortality reduction with physical activity in patients with and without cardiovascular disease. Eur Heart J. 2019;40(43):3547-55.

4. Whitaker KM, Gabriel KP, Buman MP, Pereira MA, Jacobs DR Jr, Reis JP, et al. Associations of accelerometer-measured sedentary time and physical activity with prospectively assessed Cardiometabolic risk factors: the CARDIA study. J Am Heart Assoc. 2019;8(1):e010212.

5. LaCroix AZ, Bellettiere J, Rillamas-Sun E, Di C, Evenson KR, Lewis CE, et al. Association of Light Physical Activity Measured by Accelerometry and incidence of coronary heart disease and cardiovascular disease in older women. JAMA Netw Open. 2019;2(3):e190419.

6. Ekelund U, Tarp J, Steene-Johannessen J, Hansen BH, Jefferis B, Fagerland MW, et al. Dose-response associations between accelerometry measured physical activity and sedentary time and all cause mortality: systematic review and harmonised meta-analysis. BMJ. 2019;366:14570.

7. Li X, Zhao S, Chen K, Hua W, Su Y, Yang J, et al. Dose-response association of implantable device-measured physical activity with long-term cardiac death and all-cause mortality in patients at high risk of sudden cardiac death: a cohort study. Int J Behav Nutr Phys Act. 2020;17(1):119.

8. Warren JM, Ekelund U, Besson H, Mezzani A, Geladas N, Vanhees L, et al. Assessment of physical activity - a review of methodologies with reference to epidemiological research: a report of the exercise physiology section of the European Association of Cardiovascular Prevention and Rehabilitation. Eur J Cardiovasc Prev Rehabil. 2010;17(2):127-39.
9. Cleland C, Ferguson S, Ellis G, Hunter RF. Validity of the international physical activity questionnaire (IPAQ) for assessing moderate-to-vigorous physical activity and sedentary behaviour of older adults in the United Kingdom. BMC Med Res Methodol. 2018;18(1):176.

10. Rowlands AV, Dawkins NP, Maylor B, Edwardson CL, Fairclough SJ, Davies MJ, et al. Enhancing the value of accelerometer-assessed physical activity: meaningful visual comparisons of data-driven translational accelerometer metrics. Sports Med Open. 2019;5(1):47.

11. Zhao S, Chen K, Su Y, Hua W, Chen S, Liang Z, et al. Association between patient activity and long-term cardiac death in patients with implantable cardioverter-defibrillators and cardiac resynchronization therapy defibrillators. Eur J Prev Cardiol. 2017;24(7):760-7.

12. Tornberg J, Ikäheimo TM, Kiviniemi A, Pyky R, Hautala A, Mäntysaari M, et al. Physical activity is associated with cardiac autonomic function in adolescent men. PLoS One. 2019;14(9):e0222121.

13. de Sousa TLW, Ostoli TLVDP, Sperandio EF, Arantes RL, Gagliardi ART, er al. Dose-response relationship between very vigorous physical activity and cardiovascular health assessed by heart rate variability in adults: Cross-sectional results from the EPIMOV study. PLoS One. 2019;14(1):e0210216.

14. Xhyheri B, Manfrini O, Mazzolini M, Pizzi C, Bugiardini R. Heart rate variability today. Prog Cardiovasc Dis. 2012;55(3):321-31.

15. Lahiri MK, Kannankeril PJ, Goldberger JJ. Assessment of autonomic function in cardiovascular disease: physiological basis and prognostic implications. J Am Coll Cardiol. 2008;51(18):1725-33.

16. Kleiger RE, Miller JP, Bigger JT Jr, Moss AJ. Decreased heart rate variability and its association with increased mortality after acute myocardial infarction. Am J Cardiol. 1987;59(4):256-62.

17. La Rovere MT, Bigger JT Jr, Marcus FI, Mortara A, Schwartz PJ. Baroreflex sensitivity and heart-rate variability in prediction of total cardiac mortality after myocardial infarction. ATRAMI (autonomic tone and reflexes after myocardial infarction) investigators. Lancet. 1998;351(9101):478-84.

18. Rosero SZ, Younis A, Jones P, McNitt S, Goldenberg I, Zareba W, et al. Utility of cardiovascular implantable electronic device-derived patient activity to predict clinical outcomes. Heart Rhythm. 2021;18(8):1344-51.

19. Epstein AE, DiMarco JP, Ellenbogen KA, Estes NA 3rd, Freedman RA, Gettes LS, et al. 2012 ACCF/AHA/HRS focused update incorporated into the ACCF/AHA/HRS 2008 guidelines for device-based therapy of cardiac rhythm abnormalities: a report of the American College of Cardiology Foundation/American Heart Association task force on practice guidelines and the Heart Rhythm Society. J Am Coll Cardiol. 2013;61(3):e6-75.

20. Kramer DB, Mitchell SL, Monteiro J, Jones PW, Normand SL, Hayes DL, et al. Patient activity and survival following implantable cardioverterdefibrillator implantation: the ALTITUDE activity study. J Am Heart Assoc. 2015;4(5):e001775.

21. Goto T, Mori K, Nakasuka K, Kato M, Nakayama T, Banno T, et al. Physical activity and mortality in older patients with a pacemaker. Geriatr Gerontol Int. 2020;20(2):106-11.

22. Pruvot E, Thonet G, Vesin JM. Van-Melle G, Seidl K, Schmidinger H, et al. heart rate dynamics at the onset of ventricular tachyarrhythmias as retrieved from implantable cardioverter-defibrillators in patients with coronary artery disease. Circulation. 2000;101(20):2398-404.

23. Huikuri HV, Valkama JO, Airaksinen KE, Seppänen T, Kessler KM, Takkunen $J$ T, et al. Frequency domain measures of heart rate variability before the onset of nonsustained and sustained ventricular tachycardia in patients with coronary artery disease. Circulation. 1993;87(4):1220-8.

24. Baron RM, Kenny DA. The moderator-mediator variable distinction in social psychological research: conceptual, strategic, and statistical considerations. J Pers Soc Psychol. 1986;51(6):1173-82.

25. Qiao Y, Shi R, Hou B, Wu L, Zheng L, Ding L, et al. Impact of alcohol consumption on substrate remodeling and ablation outcome of paroxysmal atrial fibrillation. J Am Heart Assoc. 2015;4(11):e002349.

26. Saunders CT, Blume JD. A classical regression framework for mediation analysis: fitting one model to estimate mediation effects. Biostatistics. 2018;19(4):514-28

27. Tofighi D, Mackinnon DP. RMediation: an R package for mediation analysis confidence intervals. Behav Res Methods. 2011;43(3):692-700.

28. Soares-Miranda L, Sattelmair J, Chaves P, Duncan GE, Siscovick DS, Stein PK, et al. Physical activity and heart rate variability in older adults: the cardiovascular health study. Circulation. 2014;129(21):2100-10. 
29. Rennie KL, Hemingway H, Kumari M, Brunner E, Malik M, Marmot M. Effects of moderate and vigorous physical activity on heart rate variability in a British study of civil servants. Am J Epidemiol. 2003;158(2):135-43.

30. Ellison GM, Waring CD, Vicinanza C, Torella D. Physiological cardiac remodelling in response to endurance exercise training: cellular and molecular mechanisms. Heart. 2012;98(1):5-10.

31. Billman GB. Cardiac autonomic neural remodeling and susceptibility to sudden cardiac death: effect of endurance exercise training. Am J Physiol Heart Circ Physiol. 2009;297(4):H1171-93.

32. Zhao S, Chen K, Su Y, Hua W, Pu J, Li H, et al. The role of variability in night-time mean heart rate on the prediction of ventricular arrhythmias and all-cause mortality in implantable cardioverter defibrillator patients. Europace. 2015;17 Suppl 2:ii76-82.

33. Tsuji H, Venditti FJ Jr, Manders ES, Evans JC, Larson MG, Feldman CL, et al. Reduced heart rate variability and mortality risk in an elderly cohort. The Framingham Heart Study Circulation. 1994;90(2):878-83.
34. Pop-Busui R, Evans GW, Gerstein HC, Fonseca V, Fleg JL, Hoogwerf BJ, et al. Effects of cardiac autonomic dysfunction on mortality risk in the action to control cardiovascular risk in diabetes (ACCORD) trial. Diabetes Care. 2010;33(7):1578-84.

35. Hull SS Jr, Vanoli E, Adamson PB, Verrier RL, Foreman RD, Schwartz PJ. Exercise training confers anticipatory protection from sudden death during acute myocardial ischemia. Circulation. 1994:89(2):548-52.

36. Nystoriak MA, Bhatnagar A. Cardiovascular effects and benefits of exercise. Front Cardiovasc Med. 2018;5:135.

\section{Publisher's Note}

Springer Nature remains neutral with regard to jurisdictional claims in published maps and institutional affiliations.
Ready to submit your research? Choose BMC and benefit from:

- fast, convenient online submission

- thorough peer review by experienced researchers in your field

- rapid publication on acceptance

- support for research data, including large and complex data types

- gold Open Access which fosters wider collaboration and increased citations

- maximum visibility for your research: over 100M website views per year

At BMC, research is always in progress.

Learn more biomedcentral.com/submissions 